\title{
Basic theorems for fuzzy differential equations in the quotient space of fuzzy numbers
}

Dong Qiu ${ }^{1,2 *}$, Chongxia Lu², Wei Zhang², Qinghua Zhang ${ }^{2}$ and Chunlai Mu

\section{"Correspondence:}

dongqiumath@163.com

'School of Computer Science,

Chongqing University, Shapingba,

Chongqing, 400044, P.R. China

${ }^{2}$ College of Mathematics and

Physics, Chongqing University of

Posts and Telecommunications,

Nanan, Chongqing, 400065,

P.R. China

Full list of author information is

available at the end of the article

\begin{abstract}
In this paper, we study the fuzzy differential equations in the quotient space of fuzzy numbers. We solve the initial value problem for fuzzy differential equations provided that the involved mappings are continuous, of uniformly bounded variation, and are bounded functions. Then we establish a variety of comparison results for the solutions of fuzzy differential equations.
\end{abstract}

MSC: $34 \mathrm{A07} ; 46540$

Keywords: fuzzy number; equivalence classes; fuzzy differential equation; comparison theorems

\section{Introduction}

Fuzzy sets were introduced in 1965 by Zadeh [1] with a view to reconcile mathematical modeling and human knowledge in the engineering science. Fuzzy set theory and its applications have been extensively developed since the 1970s and the industrial interest in fuzzy control has dramatically increased since then [2-11].

The fuzzy differential equation was first introduced by Kandel and Byatt [12]. Since then, it has been extensively investigated on the metric space $\left(E^{n}, D\right)$ of a normal fuzzy convex set with the distance $D$ given by the maximum of the Hausdorff distance between the corresponding level sets. The fuzzy differential equation was studied by Kaleva $[9,13,14]$ and Wu and Song [15-17], for the fuzzy-valued function of a real variable whose values are normal, convex, upper semicontinuous, and are compactly supported fuzzy sets in $\mathbb{R}^{n}$. Many authors studied the existence and uniqueness of solutions of the initial value problems for fuzzy differential equations under various kinds of conditions and obtained many meaningful results in [13, 18-25]. One found the local existence and uniqueness theorems for the Cauchy problem when the fuzzy-valued function $f$ satisfies the generalized Lipschitz condition [16]. The existence theorems under compactness-type conditions were studied in [17]. Based on these works, the global existence of solutions of the Cauchy problem was investigated in [15].

The above results of the fuzzy differential equation were based on the well-known and widely used Hukuhara difference, proposed by [26], and the H-differentiability of Puri and Ralescu [25], which generalized the Hukuhara differentiability of a set-valued mapping. In $[27,28]$, Mareš presented a natural equivalence relation between fuzzy quantities. This 
equivalence relation can be used to partition the set of fuzzy quantities into equivalence classes having the desired group properties under the addition operation [29-31]. Hong and Do [32] defined a more refined equivalence relation than Mareš [27] and improved Marešs results. In [33], Qiu et al. showed that the method of finding the inverse operation of fuzzy numbers in the sense of Mareš is very intuitive. The authors introduced a new concept of convergence under which the quotient space in complete. As an application of the main results, it is shown that if we identify every fuzzy number with the corresponding equivalence class, there would be more differentiable fuzzy functions than what is found in the literature. In [34] Qiu et al. further investigated the differentiability and integrability properties of such functions and gave an existence and uniqueness theorem for a solution to a fuzzy differential equation in the quotient space of fuzzy numbers.

In this paper, we will study the basic theory of fuzzy differential equations in the quotient space of fuzzy numbers. In Section 2, we recall some related concepts. In Section 3, we will solve the initial value problem for fuzzy differential equations. In Section 4, we will establish a variety of comparison results for the solutions of fuzzy differential equations, which form the essential tools for studying the fundamental theory of fuzzy differential equations. The comparison discussion shows how one can develop the theory of differential inequalities with the minimum linear structure.

\section{Preliminaries}

We start this section by recalling some pertinent concepts and key lemmas from the functions of bounded variation, fuzzy numbers, and fuzzy equivalence classes which we need for the discussion below.

Definition 2.1 [35] Let $f:[a, b] \rightarrow \mathbb{R} . f$ is said be of bounded variation if there exists a $C>0$ such that

$$
\sum_{i=1}^{n}\left|f\left(x_{i-1}\right)-f\left(x_{i}\right)\right| \leq C
$$

for every partition $a=x_{0}<x_{1}<x_{2}<\cdots<x_{n}=b$ on $[a, b]$. The set of all functions of bounded variation on $[a, b]$ is denoted by $\mathrm{BV}[a, b]$.

Definition 2.2 [35] Let $f:[a, b] \rightarrow \mathbb{R}$ be a function of bounded variation. The total variation of $f$ on $[a, b]$ is defined by

$$
V_{a}^{b}(f)=\sup _{p} \sum_{i=1}^{n}\left|f\left(x_{i-1}\right)-f\left(x_{i}\right)\right|,
$$

where $p$ represents all partitions of $[a, b]$.

Lemma 2.1 [35] For any constants $c \in \mathbb{R}$, iff $\in \mathrm{BV}[a, b]$, then so is $c f$ and

$$
V_{a}^{b}(c f)=|c| V_{a}^{b}(f)
$$

Lemma 2.2 [35] For any constants $c, d \in \mathbb{R}$, iff,$g \in \mathrm{BV}[a, b]$, then so is $c f+d g$ and

$$
V_{a}^{b}(c f+d g) \leq|c| V_{a}^{b}(f)+|d| V_{a}^{b}(g) .
$$


Lemma 2.3 [35] Let $f$ be a function of bounded variation on $[a, b]$, and let $V$ be a function defined by $V(x)=V_{x}^{b}(f)$. Then iff is continuous from the left (or right) at a point $x^{*}$, so is $V$.

Lemma 2.4 [35] If $a<b<c$, then

$$
V_{a}^{c}(f)=V_{a}^{b}(f)+V_{b}^{c}(f)
$$

Lemma 2.5 [35] Every monotonic function $f$ is of bounded variation, and $V_{a}^{b}(f)=$ $|f(a)-f(b)|$.

Lemma 2.6 [35] Iff, $g \in \mathrm{BV}[a, b]$, then so is $f g$ and

$$
V_{a}^{b}(f g) \leq c V_{a}^{b}(f)+d V_{a}^{b}(g)
$$

where $c=\sup |g(x)|<\infty, d=\sup |f(x)|<\infty$.

A fuzzy set $\tilde{x}$ of $\mathbb{R}$ is characterized by a membership function $\mu_{\tilde{x}}: \mathbb{R} \rightarrow[0,1]$. An $\alpha$ level set of $\tilde{x}$ is $[\tilde{x}]^{\alpha}=\left\{x \in \mathbb{R}: \mu_{\tilde{x}}(x) \geq \alpha\right\}$ for each $\alpha \in(0,1]$. We define the set $[\tilde{x}]^{0}$ by $[\tilde{x}]^{0}=\overline{\left\{x \in \mathbb{R}: \mu_{\tilde{x}}(x)>0\right\}}$, where $\bar{A}$ denotes the closure of a crisp set $A$. A fuzzy set $\tilde{x}$ is said to be a fuzzy number if it satisfies the following conditions [36]:

(1) $\tilde{x}$ is normal, i.e., there exists an $x_{0} \in \mathbb{R}$ such that $\mu_{\tilde{x}}\left(x_{0}\right)=1$;

(2) $\tilde{x}$ is convex, i.e., $\mu_{\tilde{x}}\left(\lambda x_{1}+(1-\lambda) x_{2}\right) \geq \min \left\{\mu_{\tilde{x}}\left(x_{1}\right), \mu_{\tilde{x}}\left(x_{2}\right)\right\}$, for all $x_{1}, x_{2} \in \mathbb{R}$ and $\lambda \in[0,1]$

(3) $\tilde{x}$ is upper semicontinuous;

(4) $[\tilde{x}]^{0}$ is compact.

Equivalently, a fuzzy number $\tilde{x}$ is a fuzzy set with nonempty bounded level sets $[\tilde{x}]^{\alpha}=$ $\left[\tilde{x}_{L}(\alpha), \tilde{x}_{R}(\alpha)\right]$ for all $\alpha \in[0,1]$, where $\left[\tilde{x}_{L}(\alpha), \tilde{x}_{R}(\alpha)\right]$ denotes a closed interval with the left end point $\tilde{x}_{L}(\alpha)$ and the right end point $\tilde{x}_{R}(\alpha)$. We denote the class of fuzzy numbers by $\mathscr{F}$. Notice that the real numbers $\mathbb{R}$ can be embedded in $\mathscr{F}$ by defining a fuzzy number $\tilde{a}$ as

$$
\mu_{\tilde{a}}(x)= \begin{cases}1, & \text { if } x=a, \\ 0, & \text { otherwise }\end{cases}
$$

for each $a \in \mathbb{R}$. Thus we will represent the singleton $\{a\}$ by $\tilde{a}$ for any real number $a \in \mathbb{R}$ and in particular $\tilde{0}$ is just the usual zero.

For any $\tilde{x}, \tilde{y} \in \mathscr{F}$ and $a \in \mathbb{R}$, owing to Zadeh's extension principle [37-39], addition and scalar multiplication are defined for any $x \in \mathbb{R}$ by

$$
\mu_{\tilde{x}+\tilde{y}}(x)=\sup _{x_{1}: x_{2}: x_{1}+x_{2}=x} \min \left\{\mu_{\tilde{x}}\left(x_{1}\right), \mu_{\tilde{y}}\left(x_{2}\right)\right\}
$$

and

$$
\mu_{a \times \tilde{x}}(x)=\mu_{a \tilde{x}}(x)= \begin{cases}\mu\left(\frac{x}{a}\right), & \text { if } a \neq 0 \\ \tilde{0}, & \text { if } a=0\end{cases}
$$


For any $\tilde{x} \in \mathscr{F}$, we define the fuzzy number $-\tilde{x} \in \mathscr{F}$ by $-\tilde{x}=(-1) \times \tilde{x}$, i.e., $\mu_{-\tilde{x}}(x)=\mu_{\tilde{x}}(-x)$, for all $x \in \mathbb{R}$. It is well known that

$$
[\tilde{x}+\tilde{y}]^{\alpha}=[\tilde{x}]^{\alpha}+[\tilde{y}]^{\alpha}=\left[\tilde{x}_{L}(\alpha)+\tilde{y}_{L}(\alpha), \tilde{x}_{R}(\alpha)+\tilde{y}_{R}(\alpha)\right]
$$

and

$$
[a \tilde{x}]^{\alpha}=a[\tilde{x}]^{\alpha}= \begin{cases}{\left[a \tilde{x}_{L}(\alpha), a \tilde{x}_{R}(\alpha)\right],} & \text { if } a>0 \\ \{0\}, & \text { if } a=0, \\ {\left[a \tilde{x}_{R}(\alpha), a \tilde{x}_{L}(\alpha)\right],} & \text { if } a<0\end{cases}
$$

for all $\tilde{x}, \tilde{y} \in \mathscr{F}$ and $a \in \mathbb{R}$. In particular, $[-\tilde{x}]^{\alpha}=-[\tilde{x}]^{\alpha}=-\left[\tilde{x}_{L}(\alpha), \tilde{x}_{R}(\alpha)\right]=\left[-\tilde{x}_{R}(\alpha),-\tilde{x}_{L}(\alpha)\right]$. By the level set representations of the fuzzy numbers $\tilde{x}, \tilde{y}, \tilde{z}$, we can get

$$
\begin{gathered}
\begin{aligned}
&\left([\tilde{x}]^{\alpha}+[\tilde{y}]^{\alpha}\right)+[\tilde{z}]^{\alpha}=[\tilde{x}]^{\alpha}+\left([\tilde{y}]^{\alpha}+[\tilde{z}]^{\alpha}\right) \\
&=\left[\tilde{x}_{L}(\alpha)+\tilde{y}_{L}(\alpha)+\tilde{z}_{L}(\alpha), \tilde{x}_{R}(\alpha)+\tilde{y}_{R}(\alpha)+\tilde{z}_{R}(\alpha)\right], \\
& {[\tilde{x}]^{\alpha}+[\tilde{y}]^{\alpha}=[\tilde{y}]^{\alpha}+[\tilde{x}]^{\alpha}=\left[\tilde{x}_{L}(\alpha)+\tilde{y}_{L}(\alpha), \tilde{x}_{R}(\alpha)+\tilde{y}_{R}(\alpha)\right], }
\end{aligned}
\end{gathered}
$$

which implies $\mathscr{F}$ is a commutative semigroup under addition. We say that a fuzzy number $\tilde{s} \in \mathscr{F}$ is symmetric [27], if

$$
\mu_{\tilde{s}}(x)=\mu_{\tilde{s}}(-x)
$$

for all $x \in \mathbb{R}$, i.e., $\tilde{s}=-\tilde{s}$. The set of all symmetric fuzzy numbers will be denoted by $\mathscr{S}$.

Definition 2.3 [32] Let $\tilde{x}, \tilde{y} \in \mathscr{F}$. We say that $\tilde{x}$ is equivalent to $\tilde{y}$ and write $\tilde{x} \sim \tilde{y}$ if and only if there exist symmetric fuzzy numbers $\tilde{s}_{1}, \tilde{s}_{2} \in \mathscr{S}$ such that

$$
\tilde{x}+\tilde{s}_{1}=\tilde{y}+\tilde{s}_{2} .
$$

The equivalence relation defined above is reflexive, symmetric, and transitive [27]. Let $\langle\tilde{x}\rangle$ denote the equivalence class containing the element $\tilde{x}$ and denote the set of equivalence classes by $\mathscr{F} / \mathscr{S}$.

Definition 2.4 [29] For a fuzzy number $\tilde{x}$, we define a function $\tilde{x}_{M}:[0,1] \rightarrow \mathbb{R}$ by assigning the midpoint of each $\alpha$-level set to $\tilde{x}_{M}(\alpha)$ for all $\alpha \in[0,1]$, i.e.,

$$
\tilde{x}_{M}(\alpha)=\frac{\tilde{x}_{L}(\alpha)+\tilde{x}_{R}(\alpha)}{2} .
$$

Then the function $\tilde{x}_{M}:[0,1] \rightarrow \mathbb{R}$ will be called the midpoint function of the fuzzy number $\tilde{x}$.

Lemma 2.7 [33] For any $\tilde{x} \in \mathscr{F}$, the midpoint function $\tilde{x}_{M}$ is continuous from the right at 0 and continuous from the left on $[0,1]$. Furthermore it is a function of bounded variation on $[0,1]$. 
It is interesting to note that the midpoint function $\tilde{x}_{M}$ is actually a gradual number and all of the gradual numbers form a group structure under addition [40, 41]. Among applications of the decomposition of a fuzzy interval using a symmetric fuzzy number and a midpoint function is the study of the fuzzy variance of a fuzzy interval $[42,43]$.

Definition 2.5 [28] Let $\tilde{x} \in \mathscr{F}$ and let $\hat{x}$ be a fuzzy number such that $\tilde{x}=\hat{x}+\tilde{s}$ for some $\tilde{s} \in \mathscr{S}$, if $\hat{x}=\tilde{y}+\tilde{s}_{1}$ for some $\tilde{y} \in \mathscr{F}$ and $\tilde{s}_{1} \in \mathscr{S}$, then $\tilde{s}_{1}=\tilde{0}$. Then the fuzzy number $\hat{x}$ will be called the Mareš core of the fuzzy number $\tilde{x}$.

In [33], Qiu et al. not only pointed out that $\tilde{x}$ is equivalent to $\tilde{y}$ if and only if they have the same midpoint function, which implies that each equivalence class corresponding to each midpoint function, but they also pointed out that for each fuzzy number $\tilde{x}$ has only one Mareš core, and $\tilde{x}$ is equivalent to $\tilde{y}$ if and only if they have the same Mareš core, which implies that all elements of an equivalence class $\langle\tilde{x}\rangle$ have the same Mareš core $\hat{x}$. Hence, the Mareš core $\hat{x}$ of $\tilde{x}$ is also called the Mareš core of the equivalence class $\langle\tilde{x}\rangle$ that contains the fuzzy number $\tilde{x}$. It is natural to define the midpoint function for an equivalence class as follows.

Definition 2.6 [34] For an equivalence class $\langle\tilde{x}\rangle \in \mathscr{F} / \mathscr{S}$, we define a midpoint function $M_{\langle\tilde{x}\rangle}:[0,1] \rightarrow \mathbb{R}$ by

$$
M_{\{\tilde{x}\rangle}(\alpha)=\hat{x}_{M}(\alpha)
$$

for all $\alpha \in[0,1]$, where $\hat{x}$ is the Mareš core of $\langle\tilde{x}\rangle$.

Definition 2.7 [34] For any $\langle\tilde{x}\rangle,\langle\tilde{y}\rangle \in \mathscr{F} / \mathscr{S}$, we define $\langle\tilde{x}\rangle+\langle\tilde{y}\rangle$ by

$$
\langle\tilde{x}\rangle+\langle\tilde{y}\rangle=\langle\tilde{x}+\tilde{y}\rangle .
$$

It is obvious that $M_{\langle\tilde{x}\rangle+\langle\tilde{y}\rangle}(\alpha)=M_{\{\tilde{x}+\tilde{y}\rangle}(\alpha)=M_{\langle\tilde{x}\rangle}(\alpha)+M_{\langle\tilde{y}\rangle}(\alpha)$, for all $\alpha \in[0,1]$. Moreover, it follows from Definitions 2.3 and 2.7 that

$$
\langle\tilde{x}\rangle+\langle\tilde{y}\rangle \supseteq\{\tilde{z}=\tilde{x}+\tilde{y}: \tilde{x} \in\langle\tilde{x}\rangle, \tilde{y} \in\langle\tilde{y}\rangle\}
$$

for any $\langle\tilde{x}\rangle,\langle\tilde{y}\rangle \in \mathscr{F} / \mathscr{S}$. In [34] Qiu et al. have given an example to show that the above inclusion can become strict.

Remark 2.1 The addition operation defined by Definition 2.7 is a group operation over the set of equivalence classes $\mathscr{F} / \mathscr{S}$ up to the equivalence relation in Definition 2.3. It means that

$$
\begin{aligned}
& \langle\tilde{x}\rangle+\langle\tilde{y}\rangle=\langle\tilde{y}\rangle+\langle\tilde{x}\rangle, \\
& (\langle\tilde{x}\rangle+\langle\tilde{y}\rangle)+\langle\tilde{z}\rangle=\langle\tilde{x}\rangle+(\langle\tilde{y}\rangle+\langle\tilde{z}\rangle), \\
& \langle\tilde{x}\rangle+\langle\tilde{y}\rangle=\langle\tilde{x}\rangle \quad \text { if and only if }\langle\tilde{y}\rangle=\langle\tilde{0}\rangle, \\
& \langle\tilde{x}\rangle+\langle\tilde{y}\rangle=\langle\tilde{0}\rangle \quad \text { if and only if }\langle\tilde{y}\rangle=\langle-\tilde{x}\rangle=-\langle\tilde{x}\rangle
\end{aligned}
$$

for any $\langle\tilde{x}\rangle,\langle\tilde{y}\rangle,\langle\tilde{z}\rangle \in \mathscr{F} \mid \mathscr{S}$. For the details of the discussion, please see $[44,45]$. 
Lemma $2.8[44,45](\mathscr{F} / \mathscr{S},+)$ is a group.

For any $\langle\tilde{x}\rangle,\langle\tilde{y}\rangle \in \mathscr{F} / \mathscr{S}$, the midpoint functions $M_{\langle\tilde{x}\rangle}$ and $M_{\{\tilde{y}\rangle}$ are continuous from the right at 0 and continuous from the left on $[0,1]$, and they are functions of bounded variation on [0,1] [33]. Then the function $M_{\langle\tilde{x}\rangle} M_{\langle\tilde{y}\rangle}$ is also continuous from the right at 0 and continuous from the left on [0,1]. In addition, by Lemma 2.6, we know that $M_{\langle\tilde{x}\rangle} M_{\langle\tilde{y}\rangle}$ is a function of bounded variation on $[0,1]$. Hence, $M_{\{\tilde{x}\rangle} M_{\{\tilde{y}\rangle}$ is the midpoint function for some fuzzy number equivalence class.

Definition 2.8 [34] Let $\langle\tilde{x}\rangle,\langle\tilde{y}\rangle,\langle\tilde{z}\rangle \in \mathscr{F} / \mathscr{S}$. If

$$
M_{\langle\tilde{z}\rangle}(\alpha)=M_{\langle\tilde{x}\rangle}(\alpha) \cdot M_{\langle\tilde{y}\rangle}(\alpha)
$$

for all $\alpha \in[0,1]$, then what we called $\langle\tilde{z}\rangle$ is the product of $\langle\tilde{x}\rangle$ and $\langle\tilde{y}\rangle$, i.e., $\langle\tilde{z}\rangle=\langle\tilde{x}\rangle \cdot\langle\tilde{y}\rangle$.

Definition 2.9 For any $\langle\tilde{x}\rangle \in \mathscr{F} \mid \mathscr{S}$ and $\lambda \in \mathbb{R}$, we define $\lambda \cdot\langle\tilde{x}\rangle=\lambda\langle\tilde{x}\rangle$ by

$$
\lambda\langle\tilde{x}\rangle=\langle\lambda \tilde{x}\rangle .
$$

It is obvious that $M_{\lambda\langle\tilde{x}\rangle}(\alpha)=M_{\langle\lambda \tilde{x}\rangle}(\alpha)=\lambda M_{\langle\tilde{x}\rangle}(\alpha)$, for all $\alpha \in[0,1]$. If $\hat{x}$ is the Mareš core of $\langle\tilde{x}\rangle$, then $\lambda \hat{x}$ is the Mareš core of $\lambda\langle\tilde{x}\rangle$. In fact, let $\hat{y}$ be the Mareš core of $\lambda\langle\tilde{x}\rangle$. Then, by Theorems 3.10 and 3.13 in [33], and Lemma 2.1, we have

$$
\begin{aligned}
& \hat{x}_{L}(\alpha)=M_{\{\tilde{x}\rangle}(\alpha)-V_{\alpha}^{1}\left(M_{\{\tilde{x}\rangle}\right), \\
& \hat{x}_{R}(\alpha)=M_{\langle\tilde{x}\rangle}(\alpha)+V_{\alpha}^{1}\left(M_{\langle\tilde{x}\rangle}\right)
\end{aligned}
$$

and

$$
\begin{aligned}
& \hat{y}_{L}(\alpha)=M_{\lambda\langle\tilde{x}\rangle}(\alpha)-V_{\alpha}^{1}\left(M_{\lambda\langle\tilde{x}\rangle}\right)=\lambda M_{\{\tilde{x}\rangle}(\alpha)-|\lambda| V_{\alpha}^{1}\left(M_{\{\tilde{x}\rangle}\right), \\
& \hat{y}_{R}(\alpha)=M_{\lambda\langle\tilde{x}\rangle}(\alpha)+V_{\alpha}^{1}\left(M_{\lambda\langle\tilde{x}\rangle}\right)=\lambda M_{\langle\tilde{x}\rangle}(\alpha)+|\lambda| V_{\alpha}^{1}\left(M_{\{\tilde{x}\rangle}\right)
\end{aligned}
$$

for all $\alpha \in[0,1]$. Hence, we get $[\hat{y}]^{\alpha}=\lambda[\hat{x}]^{\alpha}=[\lambda \hat{x}]^{\alpha}$ for all $\alpha \in[0,1]$, i.e., $\hat{y}=\lambda \hat{x}$.

Definition 2.10 Define $d_{\text {sup }}: \mathscr{F} / \mathscr{S} \times \mathscr{F} / \mathscr{S} \rightarrow \mathbb{R}^{+} \cup\{0\}$ by

$$
d_{\text {sup }}(\langle\tilde{x}\rangle,\langle\tilde{y}\rangle)=\sup _{\alpha \in[0,1]}\left|M_{\langle\tilde{x}\rangle}(\alpha)-M_{\langle\tilde{y}\rangle}(\alpha)\right|
$$

for all $\langle\tilde{x}\rangle,\langle\tilde{y}\rangle \in \mathscr{F} / \mathscr{S}$.

$\left(\mathscr{F} / \mathscr{S}, d_{\text {sup }}\right)$ is a metric space [33]. From Definition 2.10, we list here some simple properties of the metric $d_{\text {sup }}(\langle\tilde{x}\rangle,(\tilde{y}\rangle)$ :

(1) $d_{\text {sup }}(\langle\tilde{x}\rangle,\langle\tilde{z}\rangle) \leq d_{\text {sup }}(\langle\tilde{x}\rangle,\langle\tilde{y}\rangle)+d_{\text {sup }}(\langle\tilde{y}\rangle,\langle\tilde{z}\rangle)$;

(2) $d_{\text {sup }}(\lambda\langle\tilde{x}\rangle, \lambda\langle\tilde{y}\rangle)=|\lambda| d_{\text {sup }}(\langle\tilde{x}\rangle,\langle\tilde{y}\rangle)$;

(3) $d_{\text {sup }}(\langle\tilde{x}\rangle+\langle\tilde{z}\rangle,\langle\tilde{y}\rangle+\langle\tilde{z}\rangle)=d_{\text {sup }}(\langle\tilde{x}\rangle,\langle\tilde{y}\rangle)$

for all $\langle\tilde{x}\rangle,\langle\tilde{y}\rangle,\langle\tilde{z}\rangle \in \mathscr{F} \mid \mathscr{S}$ and $\lambda \in \mathbb{R}$, where the addition and scalar multiplication on $\mathscr{F} / \mathscr{S}$ are defined in Definitions 2.7 and 2.9, respectively. 


\section{Initial value problem}

In this section, we will firstly give some definitions and basic results for the fuzzy differentials and fuzzy integrals. Then we will solve the initial value problem for fuzzy differential equations.

Definition 3.1 [34] Let $T=[a, b]$. A mapping $F: T \rightarrow \mathscr{F} / \mathscr{S}$ is differentiable at $t_{0} \in T$ if there exists an $F^{\prime}\left(t_{0}\right) \in \mathscr{F} / \mathscr{S}$ such that

$$
\lim _{h \rightarrow 0} d_{\text {sup }}\left(\frac{F\left(t_{0}+h\right)-F\left(t_{0}\right)}{h}, F^{\prime}\left(t_{0}\right)\right)=0 .
$$

If $t_{0}=a\left(\right.$ or $\left.t_{0}=b\right)$, then we consider only $h \rightarrow 0^{+}$(or $\left.h \rightarrow 0^{-}\right)$. If $F, G$ are differentiable at $t$, then we have $(F+G)^{\prime}(t)=F^{\prime}(t)+G^{\prime}(t)$ and $(\lambda F)^{\prime}(t)=\lambda F^{\prime}(t), \lambda \in \mathbb{R}$.

Lemma 3.1 [34] If $F: T \rightarrow \mathscr{F} / \mathscr{S}$ is differentiable, then it is continuous with respect to $d_{\text {sup }}$.

Definition 3.2 [34] A mapping $F: T \rightarrow \mathscr{F} / \mathscr{S}$ is measurable if $F$ is measurable with respect to $d_{\text {sup }}$.

A mapping $F: T \rightarrow \mathscr{F} / \mathscr{S}$ is called integrably bounded if there exists an integrable function $h: T \rightarrow \mathbb{R}^{+} \cup\{0\}$ such that $\left|M_{F(t)}(\alpha)\right| \leq h(t)$ for all $t \in T$ and $\alpha \in[0,1]$; a mapping $F: T \rightarrow \mathscr{F} / \mathscr{S}$ is said to be of uniformly bounded variation with respect to $\alpha \in[0,1]$ (for short, uniformly bounded variation) if there exists a constant $K>0$ such that

$$
V_{0}^{1}\left(M_{F(t)}\right) \leq K
$$

for all $t \in T$ [34].

Definition 3.3 [34] Let $F: T \rightarrow \mathscr{F} / \mathscr{S}$ be measurable. The integral of $F$ over $T$, denoted $\int_{T} F(t) d t$ or $\int_{a}^{b} F(t) d t$, is a mapping $M_{\int_{T} F(t) d t}:[0,1] \rightarrow \mathbb{R}$, which is defined by the equation

$$
M_{\int_{T} F(t) d t}(\alpha)=\int_{T} M_{F(t)}(\alpha) d t
$$

for all $\alpha \in[0,1]$. The mapping $F: T \rightarrow \mathscr{F} / \mathscr{S}$ is said to be integrable over $T$ if there exists an $\left\langle\tilde{x}_{0}\right\rangle \in \mathscr{F} / \mathscr{S}$ such that $M_{\int_{T} F(t) d t}=M_{\left\{\tilde{x}_{0}\right\rangle}$. In this case, we denote the integral by

$$
\int_{T} F(t) d t=\left\langle\tilde{x}_{0}\right\rangle
$$

If $F: T \rightarrow \mathscr{F} / \mathscr{S}$ is a measurable, integrably bounded, and uniformly bounded variation of a mapping, then $F$ is integrable on $T$ and $\int_{T} F(t) d t \in \mathscr{F} / \mathscr{S}$ [34]. Also the following properties of the integral are valid. If $F, G: T \rightarrow \mathscr{F} / \mathscr{S}$ are integrable on $T$ and $\lambda \in \mathbb{R}$, then:

$$
\begin{aligned}
& \text { (1) } \int_{T}(F+G)(t) d t=\int_{T} F(t) d t+\int_{T} G(t) d t \\
& \text { (2) } \int_{T} \lambda F(t) d t=\lambda \int_{T} F(t) d t
\end{aligned}
$$


(3) $d_{\text {sup }}(F, G)$ is integrable,

(4) $\quad d_{\text {sup }}\left(\int_{T} F(t) d t, \int_{T} G(t) d t\right) \leq \int_{T} d_{\text {sup }}(F(t), G(t)) d t$,

(5) $\int_{a}^{b} F(t) d t=\int_{a}^{c} F(t) d t+\int_{c}^{b} F(t) d t, \quad a \leq c \leq b$.

Lemma 3.2 [34] Let $F: T \rightarrow \mathscr{F} / \mathscr{S}$ be continuous with respect to $d_{\text {sup }}$ and of uniformly bounded variation. Then the integral $G(t)=\int_{a}^{t} F(s)$ ds is differentiable and $G^{\prime}(t)=F(t)$ for all $t \in T$.

Lemma 3.3 [34] Let $F: T \rightarrow \mathscr{F} / \mathscr{S}$ be differentiable and the derivative $F^{\prime}$ be integrable over $T$. Then for all $t \in T$, we have $F(t)=F\left(t_{0}\right)+\int_{t_{0}}^{t} F^{\prime}(s) d s, a \leq t_{0} \leq t \leq b$.

Lemma 3.4 [34] Let $F: T \rightarrow \mathscr{F} / \mathscr{S}$ be continuous with respect to $d_{\text {sup }}$ and of uniformly bounded variation and $G(t)=\int_{a}^{t} F(s) d s$. Then for $a \leq t_{1} \leq t_{2} \leq b$ we have

$$
d_{\text {sup }}\left(G\left(t_{1}\right), G\left(t_{2}\right)\right) \leq\left(t_{2}-t_{1}\right) \sup _{t \in\left[t_{1}, t_{2}\right]} \sup _{\alpha \in[0,1]}\left|M_{F(t)}(\alpha)\right|=\left(t_{2}-t_{1}\right) \sup _{t \in\left[t_{1}, t_{2}\right]} d_{\text {sup }}(F(t),\langle\tilde{0}\rangle) .
$$

Assume that $f: T \times \mathscr{F} / \mathscr{S} \rightarrow \mathscr{F} / \mathscr{S}$ is continuous and of uniformly bounded variation. Consider the initial value problem

$$
x^{\prime}(t)=f(t, x(t)), \quad x(a)=\langle\tilde{0}\rangle .
$$

From Lemmas 3.1, 3.2, and 3.3, a lemma immediately follows.

Lemma 3.5 A mapping $x: T \rightarrow \mathscr{F} / \mathscr{S}$ is a solution to the problem (1) if and only if it is continuous, uniformly bounded variation and satisfies the integral equation

$$
x(t)=\langle\tilde{0}\rangle+\int_{a}^{t} f(s, x(s)) d s=\int_{a}^{t} f(s, x(s)) d s
$$

for all $t \in T$.

Theorem 3.1 Letf $: T \times \mathscr{F} / \mathscr{S} \rightarrow \mathscr{F} / \mathscr{S}$ be continuous with respect to $d_{\text {sup }}$ and of uniformly bounded variation on T. Suppose there exists an $L>0$ such that

$$
d_{\text {sup }}(f(t,\langle\tilde{x}\rangle),\langle\tilde{0}\rangle) \leq L
$$

for all $t \in T$ and $\langle\tilde{x}\rangle \in \mathscr{F} / \mathscr{S}$. Then the problem (1) has a solution on $T$.

Proof Since $f$ is of uniformly bounded variation, there exists a constant $K_{1}>0$ such that

$$
V_{0}^{1}\left(M_{f(t,\langle\tilde{x}))}\right) \leq K_{1}
$$

for all $t \in T$ and $\langle\tilde{x}\rangle \in \mathscr{F} / \mathscr{S}$. Let $K=(b-a) K_{1}$. Denote by $\operatorname{CBV}(T, \mathscr{F} / \mathscr{S})$ the set of all continuous and of uniformly bounded variation mappings $\varphi$ from $T$ to $\mathscr{F} / \mathscr{S}$ and 
$V_{0}^{1}\left(M_{\varphi(t)}\right) \leq K$ for all $t \in T$. We metricize $\operatorname{CBV}(T, \mathscr{F} / \mathscr{S})$ by setting

$$
D(\xi, \psi)=\sup _{T} d_{\text {sup }}(\xi(t), \psi(t))
$$

for $\xi, \psi \in \operatorname{CBV}(T, \mathscr{F} / \mathscr{S})$. Since $\left(\mathscr{F} / \mathscr{S}, d_{\text {sup }}\right)$ is a metric space, it is easy to show that also $(\mathrm{CBV}(T, \mathscr{F} / \mathscr{S}), D)$ is metric space. Next, we shall show that $(\mathrm{CBV}(T, \mathscr{F} / \mathscr{S}), D)$ is complete. By Definition 2.10, we have

$$
D(\xi, \psi)=\sup _{t \in T, \alpha \in[0,1]}\left|M_{\xi(t)}(\alpha)-M_{\psi(t)}(\alpha)\right| .
$$

Let $\left\{\xi_{n}\right\}_{n=1}^{\infty}$ is a Cauchy sequence in $\operatorname{CBV}(T, \mathscr{F} / \mathscr{S})$, i.e., for any $\varepsilon>0$, there exists a positive integer $N$ such that

$$
D\left(\xi_{m}, \xi_{n}\right)=\sup _{t \in T, \alpha \in[0,1]}\left|M_{\xi_{m}(t)}(\alpha)-M_{\xi_{n}(t)}(\alpha)\right|<\varepsilon
$$

for all $m, n>N$. Denote by $C_{1}(T \times[0,1], \mathbb{R})$ the set of all continuous function with respect to the first variable $t \in T$. Let

$$
d(x, y)=\sup _{t \in T, \alpha \in[0,1]}|x(t, \alpha)-y(t, \alpha)|
$$

for any $x, y \in C_{1}(T \times[0,1], \mathbb{R})$. Then $\left\{M_{\xi_{n}}\right\}$ is a Cauchy sequence in $C_{1}(T \times[0,1], \mathbb{R})$, which implies that for any given $t \in T$ and $\alpha \in[0,1],\left\{M_{\xi_{n}(t)}(\alpha)\right\}$ is a Cauchy sequence. Thus, there exists an $M_{\xi(t)}(\alpha)$ such that

$$
\lim _{n \rightarrow \infty}\left|M_{\xi_{n}(t)}(\alpha)-M_{\xi(t)}(\alpha)\right|=0
$$

Now we shall show that $M_{\xi(t)}(\alpha) \in C_{1}(T \times[0,1])$. Firstly, we have

$$
\left|M_{\xi_{m}(t)}(\alpha)-M_{\xi(t)}(\alpha)\right| \leq\left|M_{\xi_{m}(t)}(\alpha)-M_{\xi_{n}(t)}(\alpha)\right|+\left|M_{\xi_{n}(t)}(\alpha)-M_{\xi(t)}(\alpha)\right|
$$

for all $t \in T$ and $\alpha \in[0,1]$. Thus, letting $n \rightarrow \infty$ we have

$$
\sup _{t \in T, \alpha \in[0,1]}\left|M_{\xi_{m}(t)}(\alpha)-M_{\xi(t)}(\alpha)\right| \leq \varepsilon
$$

whenever $m>N$, which implies that $M_{\xi_{n}(t)}(\alpha)$ converges uniformly to $M_{\xi(t)}(\alpha)$ with respect to $(t, \alpha)$ on $T \times[0,1]$. Thus, we find that $M_{\xi(t)}(\alpha)$ is continuous with respect to $t \in T$, i.e., $M_{\xi(t)}(\alpha) \in C_{1}(T \times[0,1], \mathbb{R})$. For any given $\alpha_{0} \in[0,1]$, let $\left\{\alpha_{n}\right\}_{n=1}^{\infty} \subseteq[0,1]$ be a nondecreasing sequence converging to $\alpha_{0}$. For any given $t \in T$, we have

$$
\begin{aligned}
\left|M_{\xi(t)}\left(\alpha_{n}\right)-M_{\xi(t)}\left(\alpha_{0}\right)\right| \leq & \left|M_{\xi_{m}(t)}\left(\alpha_{n}\right)-M_{\xi(t)}\left(\alpha_{n}\right)\right|+\left|M_{\xi_{m}(t)}\left(\alpha_{n}\right)-M_{\xi_{m}(t)}\left(\alpha_{0}\right)\right| \\
& +\left|M_{\xi_{m}(t)}\left(\alpha_{0}\right)-M_{\xi(t)}\left(\alpha_{0}\right)\right| .
\end{aligned}
$$

Since $M_{\xi_{m}(t)}$ is continuous from the left on $[0,1]$ with respect to $\alpha$ and $M_{\xi_{m}(t)}(\alpha)$ converges uniformly to $M_{\xi(t)}(\alpha)$ with respect to $t$, we see that the limit of the right-hand side of the 
above inequality is equal to 0 as $n, m \rightarrow+\infty$. Hence $M_{\xi(t)}$ is continuous from the left on $[0,1]$ with respect to $\alpha$. Similarly, we can find that $M_{\xi(t)}$ is continuous from the right at 0 . Since $\left\{M_{\xi_{n}(t)}(\alpha)\right\}_{n=1}^{\infty}$ are of bounded variation on $[0,1]$ and converge uniformly to $M_{\xi(t)}(\alpha)$ with respect to $\alpha$, by Theorem 4.8 in [33] we obtain

$$
V_{0}^{1}\left(M_{\xi(t)}\right) \leq \lim _{n \rightarrow \infty} \inf V_{0}^{1}\left(M_{\xi_{n}(t)}\right) \leq K
$$

Hence, by Lemma 2.7, we know that $M_{\xi(t)}(\alpha)$ is a midpoint function of some fuzzy number equivalence class, we still use $\xi(t)$ to denote this fuzzy equivalence class for any $t \in T$. It is obvious that $\xi(t)$ is a mapping of uniformly bounded variation from $T$ to $\mathscr{F} / \mathscr{F}$ and for arbitrary $\varepsilon>0$ there exists a positive integer $N$ such that

$$
D\left(\xi_{n}, \xi\right)=\sup _{t \in T, \alpha \in[0,1]}\left|M_{\xi_{n}(t)}(\alpha)-M_{\xi(t)}(\alpha)\right| \leq \varepsilon / 3
$$

for all $n>N$. Since $\xi_{n}(t)$ is continuous, for any given $t_{0} \in T$ and the same $\varepsilon>0$, there exists a $\delta>0$ such that if $\left|t-t_{0}\right|<\delta$, then

$$
d_{\text {sup }}\left(\xi_{n}(t), \xi_{n}\left(t_{0}\right)\right)=\sup _{\alpha \in[0,1]}\left|M_{\xi_{n}(t)}(\alpha)-M_{\xi_{n}\left(t_{0}\right)}(\alpha)\right|<\varepsilon / 3
$$

Thus, we have

$$
\begin{aligned}
\left|M_{\xi(t)}(\alpha)-M_{\xi\left(t_{0}\right)}(\alpha)\right| \leq & \left|M_{\xi_{n}(t)}(\alpha)-M_{\xi(t)}(\alpha)\right|+\left|M_{\xi_{n}\left(t_{0}\right)}(\alpha)-M_{\xi_{n}\left(t_{0}\right)}(\alpha)\right| \\
& +\left|M_{\xi_{n}(t)}(\alpha)-M_{\xi_{n}\left(t_{0}\right)}(\alpha)\right| \\
\leq & 2 D\left(\xi_{n}, \xi\right)+d_{\text {sup }}\left(\xi_{n}(t), \xi_{n}\left(t_{0}\right)\right) \\
< & 2 \varepsilon / 3+\varepsilon / 3=\varepsilon,
\end{aligned}
$$

which implies that

$$
d_{\text {sup }}\left(\xi(t), \xi\left(t_{0}\right)\right)=\sup _{\alpha \in[0,1]}\left|M_{\xi(t)}(\alpha)-M_{\xi\left(t_{0}\right)}(\alpha)\right| \leq \varepsilon
$$

Thus, we get $\xi \in \operatorname{CBV}(T, \mathscr{F} / \mathscr{S})$. Hence, $(\operatorname{CBV}(T, \mathscr{F} / \mathscr{S}), D)$ is complete.

For $\xi \in \operatorname{CBV}(T, \mathscr{F} / \mathscr{S})$ define $G \xi$ on $T$ by the relation

$$
G \xi(t)=\langle\tilde{0}\rangle+\int_{a}^{t} f(s, \xi(s)) d s=\int_{a}^{t} f(s, \xi(s)) d s .
$$

Then for any $\alpha \in[0,1]$, we have

$$
M_{G \xi(t)}(\alpha)=M_{\int_{a}^{t} f(s, \xi(s)) d s}(\alpha)=\int_{a}^{t} M_{f(s, \xi(s))}(\alpha) d s
$$

By Lemma 2.2, we get

$$
V_{0}^{1}\left(M_{G \xi(t)}\right)=V_{0}^{1}\left(\int_{a}^{t} M_{f(s, \xi(s))} d s\right) \leq \int_{a}^{t} V_{0}^{1}\left(M_{f(s, \xi(s))}\right) d s \leq K .
$$


Thus, we see that $G \xi$ is of uniformly bounded variation. Hence, by Corollaries 4.1 and 4.3 in [34], we have $G \xi \in \operatorname{CBV}(T, \mathscr{F} / \mathscr{S})$. Let

$$
B=\{x \in \operatorname{CBV}(T, \mathscr{F} / \mathscr{S}): D(x,\langle\tilde{0}\rangle) \leq(b-a) L\} .
$$

For any $x, y \in B$ and $\lambda \in[0,1]$ :

(1) For any given $t_{0} \in T$, if $t \rightarrow t_{0}$, then

$$
\begin{aligned}
d_{\text {sup }} & \left(\lambda x(t)+(1-\lambda) y(t), \lambda x\left(t_{0}\right)+(1-\lambda) y\left(t_{0}\right)\right) \\
\leq & d_{\text {sup }}\left(\lambda x(t)+(1-\lambda) y(t), \lambda x\left(t_{0}\right)+(1-\lambda) y(t)\right) \\
\quad & +d_{\text {sup }}\left(\lambda x\left(t_{0}\right)+(1-\lambda) y(t), \lambda x\left(t_{0}\right)+(1-\lambda) y\left(t_{0}\right)\right) \\
= & d_{\text {sup }}\left(\lambda x(t), \lambda x\left(t_{0}\right)\right)+d_{\text {sup }}\left((1-\lambda) y(t),(1-\lambda) y\left(t_{0}\right)\right) \\
= & \lambda d_{\text {sup }}\left(x(t), x\left(t_{0}\right)\right)+(1-\lambda) d_{\text {sup }}\left(y(t), y\left(t_{0}\right)\right) \rightarrow 0 .
\end{aligned}
$$

(2) By Lemma 2.2 and Definitions 2.7 and 2.9, we have

$$
\begin{aligned}
V_{0}^{1}\left(M_{\lambda x(t)+(1-\lambda) y(t)}\right) & =V_{0}^{1}\left(\lambda M_{x(t)}+(1-\lambda) M_{y(t)}\right) \\
& \leq \lambda V_{0}^{1}\left(M_{x(t)}\right)+(1-\lambda) V_{0}^{1}\left(M_{y(t)}\right) \\
& \leq \lambda K+(1-\lambda) K=K .
\end{aligned}
$$

(3) By definition of $D$, we have

$$
\begin{aligned}
D(\lambda x(t)+(1-\lambda) y(t),\langle\tilde{0}\rangle) & \leq D(\lambda x(t),\langle\tilde{0}\rangle)+D(\lambda x(t)+(1-\lambda) y(t), \lambda x(t)) \\
& =D(\lambda x(t),\langle\tilde{0}\rangle)+D((1-\lambda) y(t),\langle\tilde{0}\rangle) \\
& =\lambda D(x(t),\langle\tilde{0}\rangle)+(1-\lambda) D(y(t),\langle\tilde{0}\rangle) \\
& \leq \lambda(b-a) L+(1-\lambda)(b-a) L \\
& =(b-a) L .
\end{aligned}
$$

Thus, $\lambda x(t)+(1-\lambda) y(t) \in B$, i.e., $B$ is convex subset of $\operatorname{CBV}(T, \mathscr{F} / \mathscr{S})$.

Let the set $G B=\{G x: x \in B\}$ and the sequence $\left\{\xi_{n}\right\}_{n=1}^{\infty}$ converge to $\xi$ in $B$. Since $f$ is continuous with respect to $d_{\text {sup }}$, for any $\varepsilon>0$, there exists $N>0$ such that

$$
d_{\text {sup }}\left(f\left(t, \xi_{n}(t)\right), f(t, \xi(t))\right)<\varepsilon
$$

for all $t \in T$ and $n>N$. Thus, we get

$$
\begin{aligned}
D\left(G \xi_{n}, G \xi\right) & =\sup _{t \in T} d_{\text {sup }}\left(G \xi_{n}(t), G \xi(t)\right) \\
& =\sup _{t \in T} d_{\text {sup }}\left(\int_{a}^{t} f\left(s, \xi_{n}(s)\right) d s, \int_{a}^{t} f(s, \xi(s)) d s\right) \\
& \leq \int_{a}^{b} d_{\text {sup }}\left(f\left(s, \xi_{n}(s)\right), f(s, \xi(s))\right) d s \\
& \leq(b-a) \varepsilon
\end{aligned}
$$


for all $n>N$. By Lemma 3.4, we have

$$
\begin{aligned}
d_{\text {sup }}((G x)(t),\langle\tilde{0}\rangle) & =d_{\text {sup }}((G x)(t),(G x)(a)) \\
& \leq(t-a) \sup _{T} d_{\text {sup }}(f(t,(G x)(t)),\langle\tilde{0}\rangle) \\
& \leq(b-a) L
\end{aligned}
$$

for all $x \in \operatorname{CBV}(T, \mathscr{F} / \mathscr{F})$. Thus we get

$$
D(G x,\langle\tilde{0}\rangle)=\sup _{T} d_{\text {sup }}((G x)(t),\langle\tilde{0}\rangle) \leq(b-a) L .
$$

Hence, $G$ is a continuous mapping of $B$ into itself, i.e., $G B \subseteq B$. For any $t_{1}, t_{2} \in T, t_{1} \leq t_{2}$, and $x \in B$, by Lemma 3.4, we have

$$
d_{\text {sup }}\left((G x)\left(t_{1}\right),(G x)\left(t_{1}\right)\right) \leq\left(t_{2}-t_{1}\right) \sup _{t \in\left[t_{1}, t_{2}\right]} d_{\text {sup }}(f(t,(G x)(t)),\langle\tilde{0}\rangle) \leq\left(t_{2}-t_{1}\right) L
$$

which implies that $G B$ is equicontinuous. Since $G B$ is totally bounded if and only if it is equicontinuous, we see that $G B$ is totally bounded. By Ascoli's theorem, we conclude that $G B$ is a relatively compact subset of $\operatorname{CBV}(T, \mathscr{F} / \mathscr{F})$. By Schauder's fixed point theorem, $G$ has a fixed point, and by Lemma 3.5, we know that this fixed point is a solution of (1).

Example 3.1 Define $F: T=[a, b] \rightarrow \mathscr{F} / \mathscr{S}$ by the level sets of the fuzzy mapping

$$
[\widehat{F(t)}]^{\alpha}=\left[0,2 e^{\alpha(t-a)}\right], \quad \alpha \in[0,1]
$$

where $\widehat{F(t)}$ is the Mareš core of $F(t)$, for all $t \in[a, b]$. Thus, we have

$$
M_{F(t)}(\alpha)=e^{\alpha(t-a)}
$$

for all $\alpha \in[0,1]$ and $t \in[a, b]$. It is obvious that $M_{F(t)}(\alpha)$ is continuous from the right at 0 and continuous from the left on $[0,1]$ with respect to $\alpha$. Since $M_{F(t)}(\alpha)$ is increasing with respect to $\alpha$ and by Lemma 2.5, we get

$$
V_{0}^{1}\left(M_{F(t)}\right)=e^{(t-a)}-1 \leq e^{b-a}-1 .
$$

Thus, we find that $F(t)$ is of uniformly bounded variation. Since $M_{F(t)}(\alpha)$ is uniformly continuous with respect to $t \in[a, b]$, we see that $F(t)$ is continuous with respect to $d_{\text {sup. }}$. Define $f:[a, b] \times \mathscr{F} / \mathscr{S} \rightarrow \mathscr{F} / \mathscr{S}$ by

$$
f(t,\langle\tilde{x}\rangle)=F(t) .
$$

It is obvious that $f$ is continuous with respect to $d_{\text {sup. }}$. Since

$$
M_{f(t,\{\tilde{x}))}(\alpha)=M_{F(t)}(\alpha)
$$


for all $\alpha \in[0,1]$ and by Lemma 2.6 , we get

$$
V_{0}^{1}\left(M_{f(t, \tilde{x}))}\right)=V_{0}^{1}\left(M_{F(t)}\right)=e^{(t-a)}-1 \leq e^{b-a}-1
$$

Thus, $f$ is of uniformly bounded variation. Since

$$
\begin{aligned}
d_{\text {sup }}(f(t,\langle\tilde{x}\rangle),\langle\tilde{0}\rangle) & =d_{\text {sup }}(F(t),\langle\tilde{0}\rangle)=\sup _{\alpha \in[0,1]}\left|M_{F(t)}(\alpha)-0\right| \\
& =\sup _{\alpha \in[0,1]} e^{\alpha(t-a)} \leq e^{b-a}
\end{aligned}
$$

for all $t \in[a, b]$ and $\langle\tilde{x}\rangle \in \mathscr{F} / \mathscr{S}$. Then $f(t,\langle\tilde{x}\rangle)$ satisfies the assumptions of Theorem 3.1 and hence the initial value problem

$$
x^{\prime}(t)=f(t, x(t)), \quad x(a)=\langle\tilde{0}\rangle,
$$

has a unique solution on $[a, b]$. By the proof of Theorem 3.1, we know that the unique solution $x(t)$ of the initial value problem is

$$
x(t)=\langle\tilde{0}\rangle+\int_{a}^{t} F(s) d s
$$

Then we get

$$
\begin{aligned}
M_{x(t)}(\alpha) & =M_{\langle\tilde{\langle}\rangle}(\alpha)+\int_{a}^{t} M_{F(s)}(\alpha) d s=0+\int_{a}^{t} e^{\alpha(s-a)} d s \\
& = \begin{cases}t-a, & \alpha=0, \\
\frac{1}{\alpha}\left(e^{\alpha(t-a)}-1\right), & \alpha \in(0,1] .\end{cases}
\end{aligned}
$$

Thus, we have

$$
V_{\alpha}^{1}\left(M_{x(t)}\right)=e^{t-a}-t+a-1
$$

for all $\alpha \in[0,1]$. By Theorems 3.10 and 3.13 in [33], we see that the left end points and the right end points of level sets of the Mareš core $\widehat{x(t)}$ for $x(t)$, respectively, obey

$$
\begin{aligned}
\widehat{x(t)_{L}}(\alpha) & =M_{x(t)}(\alpha)-V_{\alpha}^{1}\left(M_{x(t)}\right) \\
& = \begin{cases}2(t-a)-e^{t-a}+1, & \alpha=0, \\
\frac{1}{\alpha}\left(e^{\alpha(t-a)}-1\right)-e^{t-a}+t-a+1, & \alpha \in(0,1],\end{cases} \\
\widehat{x(t)_{R}}(\alpha) & =M_{x(t)}(\alpha)+V_{\alpha}^{1}\left(M_{x(t)}\right) \\
& = \begin{cases}e^{t-a}-1, & \alpha=0, \\
\frac{1}{\alpha}\left(e^{\alpha(t-a)}-1\right)+e^{t-a}-t+a-1, & \alpha \in(0,1] .\end{cases}
\end{aligned}
$$

\section{Comparison theorems}

Using the properties of $d_{\text {sup }}(\langle\tilde{x}\rangle,\langle\tilde{y}\rangle)$, integrals, and differential inequalities, we will establish the comparison principles in this section. 
Definition 4.1 Define $d^{+}: C[T, \mathbb{R}] \rightarrow \mathbb{R}$ by

$$
d^{+} m(t)=\varlimsup_{h \rightarrow 0^{+}} \frac{1}{h}(m(t+h)-m(t))
$$

for all $m(t) \in C[T, \mathbb{R}]$.

Theorem 4.1 Let $f: T \times \mathscr{F} / \mathscr{S} \rightarrow \mathscr{F} / \mathscr{S}$ be continuous with respect to $d_{\text {sup }}$ and of uniformly bounded variation on T. Suppose

$$
d_{\text {sup }}(f(t,\langle\tilde{x}\rangle), f(t,\langle\tilde{y}\rangle)) \leq g\left(t, d_{\text {sup }}(\langle\tilde{x}\rangle,\langle\tilde{y}\rangle)\right)
$$

for all $t \in T$ and $\langle\tilde{x}\rangle,\langle\tilde{y}\rangle \in \mathscr{F} \mid \mathscr{S}$, where $g \in C_{1}\left[T \times \mathbb{R}_{+}, \mathbb{R}_{+}\right]$and $g(t, \varphi)$ is nondecreasing with respect to $\varphi$ for all $t \in T$. Suppose the maximal solution $r(t)=r\left(t ; a, \varphi_{0}\right)$ of the scalar differential equation

$$
\frac{d \varphi}{d t}=g(t, \varphi), \quad \varphi(a)=\varphi_{0} \geq 0,
$$

exists on $T$. Then, if $x(t), y(t)$ are any two solutions of (1) through $\left(a, x_{0}\right),\left(a, y_{0}\right)$ on $T$, respectively, and $d_{\text {sup }}\left(x_{0}, y_{0}\right) \leq \varphi_{0}$ we have

$$
d_{\text {sup }}(x(t), y(t)) \leq r(t)
$$

for all $t \in T$.

Proof Let $m(t)=d_{\text {sup }}(x(t), y(t))$. Then $m\left(t_{0}\right)=d_{\text {sup }}\left(x_{0}, y_{0}\right) \leq \varphi_{0}$. Thus, we get

$$
\begin{aligned}
m(t)= & d_{\text {sup }}(x(t), y(t)) \\
= & d_{\text {sup }}\left(x_{0}+\int_{a}^{t} f(s, x(s)) d s, y_{0}+\int_{a}^{t} f(s, y(s)) d s\right) \\
\leq & d_{\text {sup }}\left(x_{0}+\int_{a}^{t} f(s, x(s)) d s, x_{0}+\int_{a}^{t} f(s, y(s)) d s\right) \\
& +d_{\text {sup }}\left(x_{0}+\int_{a}^{t} f(s, y(s)) d s, y_{0}+\int_{a}^{t} f(s, y(s)) d s\right) \\
= & d_{\text {sup }}\left(\int_{a}^{t} f(s, x(s)) d s, \int_{a}^{t} f(s, y(s)) d s\right)+d_{\text {sup }}\left(x_{0}, y_{0}\right) \\
\leq & m\left(t_{0}\right)+\int_{a}^{t} d_{\text {sup }}(f(s, x(s)), f(s, y(s))) d s \\
\leq & m\left(t_{0}\right)+\int_{a}^{t} g\left(s, d_{\text {sup }}(x(s), y(s))\right) d s \\
= & m\left(t_{0}\right)+\int_{a}^{t} g(s, m(s)) d s
\end{aligned}
$$

for all $t \in T$. By Theorem 1.9.2 in [46], we obtain $d_{\text {sup }}(x(t), y(t))=m(t) \leq r(t)$ for all $t \in T$. 
Example 4.1 Define $F: T=[a, b] \rightarrow \mathscr{F} / \mathscr{S}$ by the level sets of the fuzzy mapping

$$
[\widehat{F(t)}]^{\alpha}= \begin{cases}{\left[0,2\left(1-\alpha^{\frac{t+b-2 a}{b-a}}\right)\right],} & \text { if } \alpha \in(0,1) \\ {[0,2],} & \text { if } \alpha=0, \\ \{0\}, & \text { if } \alpha=1,\end{cases}
$$

where $\widehat{F(t)}$ is the Mareš core of $F(t)$, for all $t \in[a, b]$. Thus, we have

$$
M_{F(t)}(\alpha)= \begin{cases}1-\alpha^{\frac{t+b-2 a}{b-a},}, & \text { if } \alpha \in(0,1) \\ 1, & \text { if } \alpha=0, \\ 0, & \text { if } \alpha=1 .\end{cases}
$$

It is obvious that $F$ is continuous with respect to $d_{\text {sup }}$. Since

$$
V_{0}^{1}\left(M_{F(t)}\right)=1
$$

for all $t \in[a, b]$, we see that $F$ is of uniformly bounded variation. Define $f:[a, b] \times \mathscr{F} / \mathscr{S} \rightarrow$ $\mathscr{F} / \mathscr{S}$ by

$$
f(t,\langle\tilde{x}\rangle)=F(t)\langle\tilde{x}\rangle
$$

where the multiplication in $\mathscr{F} / \mathscr{S}$ is defined by Definition 2.6. It is obvious that $f$ is continuous with respect to $d_{\text {sup }}$ and of uniformly bounded variation. By Definition 2.10, we have

$$
\begin{aligned}
d_{\text {sup }}(f(t,\langle\tilde{x}\rangle), f(t,\langle\tilde{y}\rangle)) & =d_{\text {sup }}(F(t)\langle\tilde{x}\rangle, F(t)\langle\tilde{y}\rangle) \\
& =\sup _{\alpha \in[0,1]}\left|M_{F(t)}(\alpha) M_{\langle\tilde{x}\rangle}(\alpha)-M_{F(t)}(\alpha) M_{\tilde{y}\rangle}(\alpha)\right| \\
& \leq \sup _{\alpha \in[0,1]}\left|M_{F(t)}(\alpha)\right| d_{\text {sup }}(\langle\tilde{x}\rangle,\langle\tilde{y}\rangle) \\
& =d_{\text {sup }}(\langle\tilde{x}\rangle,\langle\tilde{y}\rangle)
\end{aligned}
$$

for all $t \in[a, b]$ and $\langle\tilde{x}\rangle,\langle\tilde{y}\rangle \in \mathscr{F} \mid \mathscr{S}$. Define the scalar differential equation

$$
\frac{d \varphi}{d t}=g(t, \varphi), \quad \varphi(a)=1
$$

where the function $g(t, \varphi)=\gamma(t) \varphi$ and $\gamma(t)=2 e^{t-a}$. It is obvious that $\gamma(t) \geq 2$ for all $t \in$ $[a, b], g \in C_{1}\left[T \times \mathbb{R}_{+}, \mathbb{R}_{+}\right]$and $g(t, \varphi)$ is nondecreasing with respect to $\varphi$ for all $t \in[a, b]$. Then

$$
g\left(t, d_{\text {sup }}(\langle\tilde{x}\rangle,\langle\tilde{y}\rangle)\right)=\gamma(t) d_{\text {sup }}(\langle\tilde{x}\rangle,\langle\tilde{y}\rangle) \geq 2 d_{\text {sup }}(\langle\tilde{x}\rangle,\langle\tilde{y}\rangle)
$$

for all $t \in[a, b]$ and $\langle\tilde{x}\rangle,\langle\tilde{y}\rangle \in \mathscr{F} \mid \mathscr{S}$. Hence, we obtain

$$
d_{\text {sup }}(f(t,\langle\tilde{x}\rangle), f(t,\langle\tilde{y}\rangle)) \leq g\left(t, d_{\text {sup }}(\langle\tilde{x}\rangle,\langle\tilde{y}\rangle)\right)
$$


for all $t \in[a, b]$ and $\langle\tilde{x}\rangle,\langle\tilde{y}\rangle \in \mathscr{F} \mid \mathscr{S}$. Let $x(a)=x_{0}$ and $y(a)=y_{0}$, such that $d_{\text {sup }}\left(x_{0}, y_{0}\right) \leq 1$. By Theorem 4.1, we conclude that

$$
d_{\text {sup }}(x(t), y(t)) \leq e^{2\left(e^{t-a}-1\right)}
$$

for all $t \in[a, b]$, where $x(t), y(t)$ are two solutions of fuzzy differential equation

$$
x^{\prime}(t)=f(t, x(t))
$$

through $\left(a, x_{0}\right),\left(a, y_{0}\right)$ on $[a, b]$.

Corollary 4.1 Let $f: T \times \mathscr{F} / \mathscr{S} \rightarrow \mathscr{F} / \mathscr{S}$ be continuous with respect to $d_{\text {sup }}$ and of uniformly bounded variation on $T$. Suppose

$$
d_{\text {sup }}(f(t,\langle\tilde{x}\rangle),\langle\tilde{0}\rangle) \leq g\left(t, d_{\text {sup }}(\langle\tilde{x}\rangle,\langle\tilde{0}\rangle)\right)
$$

for all $t \in T$ and $\langle\tilde{x}\rangle \in \mathscr{F} \mid \mathscr{S}$, where $g$ satisfies the assumptions of Theorem 4.1. Then, if $d_{\text {sup }}\left(x_{0},\langle\tilde{0}\rangle\right) \leq \varphi_{0}$, we have

$$
d_{\text {sup }}(x(t),\langle\tilde{0}\rangle) \leq r(t)
$$

for all $t \in T$, where $x(t)$ is any solution of (1) through $\left(a, x_{0}\right)$ on $T$ and $r(t)=r\left(t, a, \varphi_{0}\right)$ is the maximal solution of the scalar differential equation (2) on $T$.

Proof In fact, we can show this corollary by a similar method to Theorem 4.1.

Theorem 4.2 Let $f: T \times \mathscr{F} / \mathscr{S} \rightarrow \mathscr{F} / \mathscr{S}$ be continuous with respect to $d_{\text {sup }}$ and of uniformly bounded variation on T. Suppose

$$
d_{\text {sup }}(f(t,\langle\tilde{x}\rangle), f(t,\langle\tilde{y}\rangle)) \leq g\left(t, d_{\text {sup }}(\langle\tilde{x}\rangle,\langle\tilde{y}\rangle)\right)
$$

for all $t \in T$ and $\langle\tilde{x}\rangle,\langle\tilde{y}\rangle \in \mathscr{F} \mid \mathscr{S}$, where $g \in C_{1}\left[T \times \mathbb{R}_{+}, \mathbb{R}_{+}\right]$. Suppose the maximal solution $r(t)=r\left(t, a, \varphi_{0}\right)$ of the scalar differential equation (2) exists on $T$. Then, if $x(t), y(t)$ are any two solutions of (1) through $\left(a, x_{0}\right),\left(a, y_{0}\right)$ on $T$, respectively, and $d_{\text {sup }}\left(x_{0}, y_{0}\right) \leq \varphi_{0}$ we have

$$
d_{\text {sup }}(x(t), y(t)) \leq r(t)
$$

for all $t \in T$.

Proof Let $m(t)=d_{\text {sup }}(x(t), y(t))$. Then $m\left(t_{0}\right)=d_{\text {sup }}\left(x_{0}, y_{0}\right) \leq \varphi_{0}$ and for any fixed $t \in T$ and $h \neq 0$ with $t+h \in T$, we have

$$
m(t+h)-m(t)=d_{\text {sup }}(x(t+h), y(t+h))-d_{\text {sup }}(x(t), y(t)) .
$$

Since

$$
\begin{aligned}
d_{\text {sup }}(x(t+h), y(t+h)) \leq & d_{\text {sup }}(x(t+h), x(t)+h f(t, x(t))) \\
& +d_{\text {sup }}(x(t)+h f(t, x(t)), y(t+h)),
\end{aligned}
$$




$$
\begin{aligned}
d_{\text {sup }}(x(t)+h f(t, x(t)), y(t+h)) \leq & d_{\text {sup }}(y(t)+h f(t, y(t)), y(t+h)) \\
& +d_{\text {sup }}(x(t)+h f(t, x(t)), y(t)+h f(t, y(t)))
\end{aligned}
$$

and

$$
\begin{aligned}
& d_{\text {sup }}(x(t)+h f(t, x(t)), y(t)+h f(t, y(t))) \\
& \leq d_{\text {sup }}(x(t)+h f(t, x(t)), x(t)+h f(t, y(t))) \\
& \quad+d_{\text {sup }}(x(t)+h f(t, y(t)), y(t)+h f(t, y(t))) \\
&=|h| d_{\text {sup }}(f(t, x(t)), f(t, y(t)))+d_{\text {sup }}(x(t), y(t)),
\end{aligned}
$$

we get

$$
\begin{aligned}
\frac{m(t+h)-m(t)}{h} \leq & \frac{1}{h} d_{\text {sup }}(x(t+h), x(t)+h f(t, x(t))) \\
& +\frac{1}{h} d_{\text {sup }}(y(t)+h f(t, y(t)), y(t+h)) \\
& +\frac{|h|}{h} d_{\text {sup }}(f(t, x(t)), f(t, y(t))) .
\end{aligned}
$$

Thus, by Definition 4.1, we get

$$
\begin{aligned}
d^{+} m(t)= & \varlimsup_{h \rightarrow 0^{+}} \frac{1}{h}(m(t+h)-m(t)) \\
\leq & \varlimsup_{h \rightarrow 0^{+}} d_{\sup }\left(\frac{x(t+h)-x(t)}{h}, f(t, x(t))\right) \\
& +\varlimsup_{h \rightarrow 0^{+}} d_{\sup }\left(f(t, y(t)), \frac{y(t+h)-y(t)}{h}\right)+d_{\text {sup }}(f(t, x(t)), f(t, y(t))) \\
= & d_{\sup }(f(t, x(t)), f(t, y(t))) \leq g\left(t, d_{\text {sup }}(x(t), y(t))\right)=g(t, m(t)) .
\end{aligned}
$$

By Theorem 1.4.1 in [46], we obtain $d_{\text {sup }}(x(t), y(t))=m(t) \leq r(t)$, for all $t \in T$.

Corollary 4.2 Let $f: T \times \mathscr{F} / \mathscr{S} \rightarrow \mathscr{F} / \mathscr{S}$ be continuous with respect to $d_{\text {sup }}$ and of uniformly bounded variation on $T$. If

$$
d_{\text {sup }}(f(t,\langle\tilde{x}\rangle),\langle\tilde{0}\rangle) \leq g\left(t, d_{\text {sup }}(\langle\tilde{x}\rangle,\langle\tilde{0}\rangle)\right)
$$

for all $t \in T$ and $\langle\tilde{x}\rangle \in \mathscr{F} / \mathscr{S}$, where $g$ satisfies the assumptions of Theorem 4.2 , then, if $d_{\text {sup }}\left(x_{0},\langle\tilde{0}\rangle\right) \leq \varphi_{0}$, we have

$$
d_{\text {sup }}(x(t),\langle\tilde{0}\rangle) \leq r(t)
$$

for all $t \in T$, where $x(t)$ is any solution of (1) through $\left(a, x_{0}\right)$ on $T$ and $r(t)=r\left(t, a, \varphi_{0}\right)$ is the maximal solution of the scalar differential equation (2) on $T$.

Proof In fact, we can show this corollary by a similar method to Theorem 4.2. 
Theorem 4.3 Let $f: T \times \mathscr{F} / \mathscr{S} \rightarrow \mathscr{F} / \mathscr{S}$ be continuous with respect to $d_{\text {sup }}$ and of uniformly bounded variation on T. Suppose

$$
\varlimsup_{h \rightarrow 0^{+}} \frac{1}{h}\left\{d_{\text {sup }}(\langle\tilde{x}\rangle+h f(t,\langle\tilde{x}\rangle),,\langle\tilde{y}\rangle+h f(t,\langle\tilde{y}\rangle))-d_{\text {sup }}(\langle\tilde{x}\rangle,\langle\tilde{y}\rangle)\right\} \leq g\left(t, d_{\text {sup }}(\langle\tilde{x}\rangle,\langle\tilde{y}\rangle)\right)
$$

for all $t \in T$ and $\langle\tilde{x}\rangle,\langle\tilde{y}\rangle \in \mathscr{F} \mid \mathscr{S}$, where $g \in C_{1}\left[T \times \mathbb{R}_{+}, \mathbb{R}\right]$. Suppose the maximal solution $r(t)=r\left(t, a, \varphi_{0}\right)$ of the scalar differential equation (2) exists on $T$. Then, if $x(t), y(t)$ are any two solutions of (1) through $\left(a, x_{0}\right),\left(a, y_{0}\right)$ on $T$, respectively, and $d_{\text {sup }}\left(x_{0}, y_{0}\right) \leq \varphi_{0}$, we have

$$
d_{\text {sup }}(x(t), y(t)) \leq r(t)
$$

for all $t \in T$.

Proof Let $m(t)=d_{\text {sup }}(x(t), y(t))$. Then $m\left(t_{0}\right)=d_{\text {sup }}\left(x_{0}, y_{0}\right) \leq \varphi_{0}$ and for any fixed $t \in T$ and $h \neq 0$ with $t+h \in T$, from the proof of Theorem 4.2, we have

$$
\begin{aligned}
m(t+h)-m(t)= & d_{\text {sup }}(x(t+h), y(t+h))-d_{\text {sup }}(x(t), y(t)) \\
\leq & d_{\text {sup }}(x(t+h), x(t)+h f(t, x(t)))+d_{\text {sup }}(y(t)+h f(t, y(t)), y(t+h)) \\
& +d_{\text {sup }}(x(t)+h f(t, x(t)), y(t)+h f(t, y(t)))-d_{\text {sup }}(x(t), y(t)),
\end{aligned}
$$

which implies that

$$
\begin{aligned}
d^{+} m(t)= & \varlimsup_{h \rightarrow 0^{+}} \frac{1}{h}(m(t+h)-m(t)) \\
\leq & \varlimsup_{h \rightarrow 0^{+}} d_{\text {sup }}\left(\frac{x(t+h)-x(t)}{h}, f(t, x(t))\right)+\varlimsup_{h \rightarrow 0^{+}} d_{\text {sup }}\left(f(t, y(t)), \frac{y(t+h)-y(t)}{h}\right) \\
& +\varlimsup_{h \rightarrow 0^{+}} \frac{1}{h}\left\{d_{\text {sup }}(x(t)+h f(t, x(t)), y(t)+h f(t, y(t)))-d_{\text {sup }}(x(t), y(t))\right\} \\
= & \varlimsup_{h \rightarrow 0^{+}} \frac{1}{h}\left\{d_{\text {sup }}(x(t)+h f(t, x(t)), y(t)+h f(t, y(t)))-d_{\text {sup }}(x(t), y(t))\right\} \\
\leq & g\left(t, d_{\text {sup }}(x(t), y(t))\right)=g(t, m(t)) .
\end{aligned}
$$

By Theorem 1.4.1 in [46], we obtain $d_{\text {sup }}(x(t), y(t))=m(t) \leq r(t)$, for all $t \in T$.

Corollary 4.3 Let $f: T \times \mathscr{F} / \mathscr{S} \rightarrow \mathscr{F} / \mathscr{S}$ be continuous with respect to $d_{\text {sup }}$ and of uniformly bounded variation on T. Suppose

$$
\varlimsup_{h \rightarrow 0^{+}} \frac{1}{h}\left\{d_{\text {sup }}(\langle\tilde{x}\rangle+h f(t,\langle\tilde{x}\rangle),\langle\tilde{0}\rangle)-d_{\text {sup }}(\langle\tilde{x}\rangle,\langle\tilde{0}\rangle)\right\} \leq g\left(t, d_{\text {sup }}(\langle\tilde{x}\rangle,\langle\tilde{0}\rangle)\right)
$$

for all $t \in T$ and $\langle\tilde{x}\rangle \in \mathscr{F} / \mathscr{S}$, where $g$ satisfies the assumptions of Theorem 4.3. Then, if $d_{\text {sup }}\left(x_{0},\langle\tilde{0}\rangle\right) \leq \varphi_{0}$, we have

$$
d_{\text {sup }}(x(t),\langle\tilde{0}\rangle) \leq r(t)
$$

for all $t \in T$, where $x(t)$ is any solution of (1) through $\left(a, x_{0}\right)$ on $T$ and $r(t)=r\left(t ; a, \varphi_{0}\right)$ is the maximal solution of the scalar differential equation (2) on $T$. 
Proof In fact, we can show this corollary by a similar method to Theorem 4.3.

Example 4.2 Define two fuzzy mappings $F, G:[a, b] \rightarrow \mathscr{F} / \mathscr{S}$ by the level sets

$$
[\widehat{F(t)}]^{\alpha}=\left[-e^{\frac{1}{2+\alpha}(b-a)\left(\frac{t-a}{b-a}\right)^{2+\alpha}}, 0\right]
$$

and

$$
[\widehat{G(t)}]^{\alpha}=\left[0,\left(\frac{t-a}{b-a}\right)^{1+\alpha}\right]
$$

for all $\alpha \in[0,1]$, where $\widehat{F(t)}$ and $\widehat{G(t)}$ are the Mareš core of $F(t)$ and $G(t)$, respectively, for all $t \in[a, b]$. Thus, we have

$$
M_{F(t)}(\alpha)=-\frac{1}{2} e^{\frac{1}{2+\alpha}(b-a)\left(\frac{t-a}{b-a}\right)^{2+\alpha}} \text { and } \quad M_{G(t)}(\alpha)=\frac{1}{2}\left(\frac{t-a}{b-a}\right)^{1+\alpha}
$$

for all $\alpha \in[0,1]$ and $t \in[a, b]$. It is obvious that $M_{F(t)}(\alpha)$ and $M_{G(t)}(\alpha)$ are continuous from the right at 0 and continuous from the left on $[0,1]$ with respect to $\alpha$. Since $M_{F(t)}(\alpha)$ and $M_{G(t)}(\alpha)$ are decreasing with respect to $\alpha$ and by Lemma 2.4, we get

$$
V_{0}^{1}\left(M_{F(t)}\right)=\frac{1}{2}\left(e^{\frac{1}{2}(b-a)\left(\frac{t-a}{b-a}\right)^{2}}-e^{\frac{1}{3}(b-a)\left(\frac{t-a}{b-a}\right)^{3}}\right) \leq \frac{1}{2} e^{\frac{1}{2}(b-a)\left(\frac{t-a}{b-a}\right)^{2}} \leq \frac{1}{2} e^{\frac{1}{2}(b-a)}
$$

and

$$
V_{0}^{1}\left(M_{G(t)}\right)=\frac{1}{2}\left(\left(\frac{t-a}{b-a}\right)^{1}-\left(\frac{t-a}{b-a}\right)^{2}\right)=-\frac{1}{2}\left(\frac{t-a}{b-a}-\frac{1}{2}\right)^{2}+\frac{1}{8} \leq \frac{1}{8} .
$$

Thus, we see that $F(t)$ and $G(t)$ are of uniformly bounded variation. Since $M_{F(t)}(\alpha)$ and $M_{G(t)}(\alpha)$ are uniformly continuous with respect to $t \in[a, b]$, we see that $F(t)$ and $G(t)$ are continuous with respect to $d_{\text {sup. }}$. Define $f:[a, b] \times \mathscr{F} / \mathscr{S} \rightarrow \mathscr{F} / \mathscr{S}$ by

$$
f(t,\langle\tilde{x}\rangle)=F(t)\langle\tilde{x}\rangle+G(t)
$$

where the addition and multiplication in $\mathscr{F} / \mathscr{S}$ are defined by Definitions 2.7 and 2.8, respectively. It is obvious that $f$ is continuous with respect to $d_{\text {sup }}$ and of uniformly bounded variation. By Definition 2.10, we get

$$
\begin{aligned}
d_{\text {sup }} & (\langle\tilde{x}\rangle+h f(t,\langle\tilde{x}\rangle),\langle\tilde{y}\rangle+h f(t,\langle\tilde{y}\rangle))-d_{\text {sup }}(\langle\tilde{x}\rangle,\langle\tilde{y}\rangle) \\
= & d_{\text {sup }}(\langle\tilde{x}\rangle+h(F(t)\langle\tilde{x}\rangle+G(t)),\langle\tilde{y}\rangle+h(F(t)\langle\tilde{y}\rangle+G(t)))-d_{\text {sup }}(\langle\tilde{x}\rangle,\langle\tilde{y}\rangle) \\
= & d_{\text {sup }}(\langle\tilde{x}\rangle+h F(t)\langle\tilde{x}\rangle,\langle\tilde{y}\rangle+h F(t)\langle\tilde{y}\rangle)-d_{\text {sup }}(\langle\tilde{x}\rangle,\langle\tilde{y}\rangle) \\
= & \sup _{\alpha \in[0,1]}\left|M_{\langle\tilde{x}\rangle}(\alpha)+h M_{F(t)}(\alpha) M_{\langle\tilde{x}\rangle}(\alpha)-M_{\langle\tilde{y}\rangle}(\alpha)-h M_{F(t)}(\alpha) M_{\langle\tilde{y}\rangle}(\alpha)\right|-d_{\text {sup }}(\langle\tilde{x}\rangle,\langle\tilde{y}\rangle) \\
\leq & \left(1+h \sup _{\alpha \in[0,1]} M_{F(t)}(\alpha)\right) d_{\text {sup }}(\langle\tilde{x}\rangle,\langle\tilde{y}\rangle)-d_{\text {sup }}(\langle\tilde{x}\rangle,\langle\tilde{y}\rangle) \\
= & h \sup _{\alpha \in[0,1]} M_{F(t)}(\alpha) d_{\text {sup }}(\langle\tilde{x}\rangle,\langle\tilde{y}\rangle)
\end{aligned}
$$




$$
\begin{aligned}
& =-\frac{1}{2} h e^{\frac{1}{3}(b-a)\left(\frac{t-a}{b-a}\right)^{3}} d_{\text {sup }}(\langle\tilde{x}\rangle,\langle\tilde{y}\rangle) \\
& \leq-\frac{1}{2} h d_{\text {sup }}(\langle\tilde{x}\rangle,\langle\tilde{y}\rangle)
\end{aligned}
$$

for all $h>0$ sufficiently small. Hence

$$
\varlimsup_{h \rightarrow 0^{+}} \frac{1}{h}\left\{d_{\text {sup }}(\langle\tilde{x}\rangle+h f(t,\langle\tilde{x}\rangle),\langle\tilde{y}\rangle+h f(t,\langle\tilde{y}\rangle))-d_{\text {sup }}(\langle\tilde{x}\rangle,\langle\tilde{y}\rangle)\right\} \leq-\frac{1}{2} d_{\text {sup }}(\langle\tilde{x}\rangle,\langle\tilde{y}\rangle)
$$

for all $t \in[a, b]$ and $\langle\tilde{x}\rangle,\langle\tilde{y}\rangle \in \mathscr{F} \mid \mathscr{S}$. Define the scalar differential equation

$$
\frac{d \varphi}{d t}=g(t, \varphi), \quad \varphi(a)=1
$$

where the function $g(t, \varphi)=\gamma(t) \varphi$ and $\gamma(t)=-1 / 2 e^{a-t}$. It is obvious that $\gamma(t) \geq-1 / 2$ for all $t \in[a, b], g \in C_{1}\left[[a, b] \times \mathbb{R}_{+}, \mathbb{R}\right]$. Then

$$
g\left(t, d_{\text {sup }}(\langle\tilde{x}\rangle,\langle\tilde{y}\rangle)\right)=\gamma(t) d_{\text {sup }}(\langle\tilde{x}\rangle,\langle\tilde{y}\rangle) \geq-\frac{1}{2} d_{\text {sup }}(\langle\tilde{x}\rangle,\langle\tilde{y}\rangle)
$$

for all $t \in[a, b]$ and $\langle\tilde{x}\rangle,\langle\tilde{y}\rangle \in \mathscr{F} / \mathscr{S}$. Hence, we obtain

$$
\varlimsup_{h \rightarrow 0^{+}} \frac{1}{h}\left\{d_{\text {sup }}(\langle\tilde{x}\rangle+h f(t,\langle\tilde{x}\rangle),\langle\tilde{y}\rangle+h f(t,\langle\tilde{y}\rangle))-d_{\text {sup }}(\langle\tilde{x}\rangle,\langle\tilde{y}\rangle)\right\} \leq g\left(t, d_{\text {sup }}(\langle\tilde{x}\rangle,\langle\tilde{y}\rangle)\right)
$$

for all $t \in[a, b]$ and $\langle\tilde{x}\rangle,\langle\tilde{y}\rangle \in \mathscr{F} / \mathscr{S}$. Let $x(a)=x_{0}$ and $y(a)=y_{0}$, such that $d_{\text {sup }}\left(x_{0}, y_{0}\right) \leq 1$. By Theorem 4.3, we conclude that

$$
d_{\text {sup }}(x(t), y(t)) \leq e^{\frac{1}{2}\left(e^{a-t}-1\right)}
$$

for all $t \in[a, b]$, where $x(t), y(t)$ are two solutions of the fuzzy differential equation

$$
x^{\prime}(t)=f(t, x(t))
$$

through $\left(a, x_{0}\right),\left(a, y_{0}\right)$ on $[a, b]$.

\section{Conclusions}

In this paper, we have researched the basic theory of fuzzy differential equations in the quotient space of fuzzy numbers. We have solved the initial value problem for the fuzzy differential equations provided that $f$ is a continuous with respect to $d_{\text {sup }}$, of uniformly bounded variation on $T$, and is a bounded function, and then we have established a variety of comparison results for the solutions of fuzzy differential equations which form the essential tools for studying the fundamental theory of fuzzy differential equations. The comparison discussion shows how, with the minimum linear structure, one can develop the theory of differential inequalities that are important in comparison principles. We also hope that our results in this paper may lead to significant, new, and innovative results in related fields. 
Authors' contributions

All authors contributed equally to the writing of this paper. All authors read and approved the final manuscript.

\section{Author details}

${ }^{1}$ School of Computer Science, Chongqing University, Shapingba, Chongqing, 400044, P.R. China. ${ }^{2}$ College of Mathematics and Physics, Chongqing University of Posts and Telecommunications, Nanan, Chongqing, 400065, P.R. China. ${ }^{3}$ School of Mathematics and Statistics, Chongqing University, Shapingba, Chongqing, 400044, P.R. China.

\section{Acknowledgements}

This work was supported by The National Natural Science Foundations of China (Grant Nos. 11201512 and 61472056) and The Natural Science Foundation Project of CQ CSTC (cstc2012jjA00001).

\section{Received: 24 June 2014 Accepted: 19 November 2014 Published: 02 Dec 2014}

\section{References}

1. Zadeh, LA: Fuzzy sets. Inf. Control 8, 338-353 (1965)

2. Chalco-Cano, Y, Román-Flores, H: Some remarks on fuzzy differential equations via differential inclusions. Fuzzy Sets Syst. 230, 3-20 (2013)

3. Ding, Z, Shen, $H$, Kandel, A: Hypergraph partitioning for the parallel computing of fuzzy differential equations. Fuzzy Sets Syst. 230, 142-161 (2013)

4. Dizicheh, AK, Salahshour, S, Ismail, FB: A note on 'Numerical solutions of fuzzy differential equations by extended Runge-Kutta-like formulae of order 4'. Fuzzy Sets Syst. 233, 96-100 (2013)

5. Drainkov, D, Hellendoom, H, Reinfrank, M: An Introduction to Fuzzy Control. Springer, Berlin (1996)

6. Dubois, D, Prade, H: Towards fuzzy differential calculus, part 1: integration of fuzzy mappings. Fuzzy Sets Syst. 8, 1-17 (1982)

7. Dubois, D, Prade, H: Towards fuzzy differential calculus, part 2: integration on fuzzy intervals. Fuzzy Sets Syst. 8, 105-116 (1982)

8. Dubois, D, Prade, H: Towards fuzzy differential calculus, part 3: differentiation. Fuzzy Sets Syst. 8, 225-234 (1982)

9. Kaleva, O: Fuzzy differential equation. Fuzzy Sets Syst. 24, 301-317 (1987)

10. Lakshmikantham, V, Mohapatra, RN: Theory of Fuzzy Differential Equations and Inclusions. Academic Press, New York (2003)

11. Novak, V: Fuzzy Sets and Their Applications. Hilger, Bristol (1988)

12. Kandel, A, Byatt, WJ: Fuzzy differential equations. In: Proceedings of International Conference on Cybernetics and Society, Tokyo (1978)

13. Kaleva, O: The Cauchy problem for fuzzy differential equations. Fuzzy Sets Syst. 35, 389-396 (1990)

14. Kaleva, O: On the calculus of fuzzy valued mappings. Appl. Math. Lett. 3, 55-59 (1990)

15. Song, SJ: Global existence of solutions to fuzzy differential equation. Fuzzy Sets Syst. 115, 371-376 (2000)

16. Wu, CX, Song, SJ, Lee, ES: Approximate solutions, existence and uniqueness of the Cauchy problem of fuzzy differential equations. J. Math. Anal. Appl. 202, 629-644 (1996)

17. Wu, CX, Song, SJ: Existence theorem of fuzzy differential equations under compactness-type condition. Inf. Sci. 108, 123-134 (1998)

18. Buckley, JJ, Feuring, TH: Fuzzy differential equations. Fuzzy Sets Syst. 110, 43-54 (2000)

19. Ding, Z, Ming, M, Kandel, A: Existence of solutions of fuzzy differential equations. Inf. Sci. 99, 205-217 (1997)

20. Gasilov, N, Amrahov, SE, Fatullayev, AG: Solution of linear differential equations with fuzzy boundary values. Fuzzy Sets Syst. 257, 169-183 (2014)

21. Karpenko, D, Gorder, RAV, Kandel, A: The Cauchy problem for complex fuzzy differential equations. Fuzzy Sets Syst. 245, 18-29 (2014)

22. Lakshmikantham, $\vee$, Mohapatra, RN: Basic properties of solutions of fuzzy differential equations. Nonlinear Stud. 8, 113-124 (2000)

23. Li, JX, Zhao, AM, Yan, JR: The Cauchy problem of fuzzy differential equations under generalized differentiability. Fuzzy Sets Syst. 200, 1-24 (2012)

24. Nieto, JJ: The Cauchy problem for continuous fuzzy differential equations. Fuzzy Sets Syst. 102, 113-124 (1999)

25. Puri, ML, Ralescu, DA: Differentials of fuzzy functions. J. Math. Anal. Appl. 91, 552-558 (1983)

26. Hukuhara, M: Intégration des applications mesurables dont la valeur est un compact convexe. Funkc. Ekvacioj 10 , 205-223 (1967)

27. Mareš, M: Addition of fuzzy quantities: disjunction-conjunction approach. Kybernetika 25, 104-116 (1989)

28. Mareš, M: Additive decomposition of fuzzy quantities. Fuzzy Sets Syst. 47, 341-346 (1992)

29. Jamison, KD: A normed space of fuzzy number equivalence classes. UCD/CCM Report No. 112, October (1997)

30. Panda, G, Panigrahi, M, Nanda, S: Equivalence class in the set of fuzzy numbers and its application in decision-making problems. Int. J. Math. Math. Sci. 35, 741-765 (2006)

31. Wu, CX, Zhao, Z: Some notes on the characterization of compact sets of fuzzy sets with $L_{p}$ metric. Fuzzy Sets Syst. $159,2104-2115(2008)$

32. Hong, D, Do, H: Additive decomposition of fuzzy quantities. Inf. Sci. 88, 201-207 (1996)

33. Qiu, D, Lu, CX, Zhang, W, Lan, YY: Algebraic properties and topological properties of the quotient space of fuzzy numbers based on Mareš equivalence relation. Fuzzy Sets Syst. 245, 63-82 (2014)

34. Qiu, D, Zhang, W, Lu, CX: On fuzzy differential equations in the quotient space of fuzzy numbers. Fuzzy Sets Syst. (2014, submitted)

35. Kolmogorov, AN, Fomin, SV: Introductory Real Analysis. Dover, New York (1975)

36. Dubois, D, Prade, H: Fuzzy Sets and Systems. Academic Press, New York (1980)

37. Zadeh, LA: The concept of a linguistic variable and its application to approximate reasoning - I. Inf. Sci. 8, 199-251 (1975)

38. Zadeh, LA: The concept of a linguistic variable and its application to approximate reasoning - II. Inf. Sci. 8, 301-357 (1975) 
39. Zadeh, LA: The concept of a linguistic variable and its application to approximate reasoning - III. Inf. Sci. 9, 43-80 (1975)

40. Dubois, D, Prade, H: Gradual elements in a fuzzy set. Soft Comput. 12, 165-175 (2008)

41. Fortin, J, Dubois, D, Fargier, H: Gradual numbers and their application to fuzzy interval analysis. IEEE Trans. Fuzzy Syst. $16,388-402(2008)$

42. Dubois, D, Fargier, H, Fortin, J: The empirical variance of a set of fuzzy intervals. In: Proc. of the 2005 IEEE Int. Conf. on Fuzzy Systems (FUZZ-IEEE 2005), Reno, Nevada, 22-25 May, pp. 885-890. IEEE Press, New York (2005)

43. Dubois, D, Kerre, E, Mesiar, R, Prade, H: Fuzzy interval analysis. In: Dubois, D, Prade, H (eds.) Fundamentals of Fuzzy Sets. The Handbooks of Fuzzy Sets, pp. 483-581. Kluwer Academic, Boston (2000)

44. Jamison, KD: Modeling uncertainty using probabilistic based possibility theory with applications to optimization. Doctoral thesis, University of Colorado at Denver (1998)

45. Jamison, KD: Possibility as cumulative probability and a normed space of congruence classes of fuzzy numbers motivated by an expected utility functional. Fuzzy Sets Syst. 111, 331-339 (2000)

46. Lakshmikantham, V, Leela, S: Differential and Integral Inequalities, vol. I. Academic Press, New York (1996)

10.1186/1687-1847-2014-303

Cite this article as: Qiu et al.: Basic theorems for fuzzy differential equations in the quotient space of fuzzy numbers. Advances in Difference Equations 2014, 2014:303

\section{Submit your manuscript to a SpringerOpen ${ }^{\circ}$ journal and benefit from:}

- Convenient online submission

Rigorous peer review

- Immediate publication on acceptance

- Open access: articles freely available online

- High visibility within the field

- Retaining the copyright to your article 